\title{
Patrones de prescripción de antidiabéticos en un grupo de pacientes colombianos
}

\author{
Jorge Enrique Machado Alba, ${ }^{1}$ Juan Carlos Moncada Escobar ${ }^{2}$ y \\ Giovanny Mesa Escobar ${ }^{2}$
}

Forma de citar

Machado Alba JE, Moncada Escobar JC, Mesa Escobar G. Patrones de prescripción de antidiabéticos en un grupo de pacientes colombianos. Rev Panam Salud Pública. 2007;22(2):124-31.

RESUMEN Objetivos. Determinar los patrones de prescripción de medicamentos antidiabéticos en un grupo de personas afiliadas al Sistema General de Seguridad Social en Salud (SGSSS) de Colombia.

Métodos. Se incluyeron en el estudio 7308 afiliados al SGSSS con diabetes de tipo 1 ó 2, de ambos sexos y de todas las edades, tratados durante por lo menos tres meses (de mayo a julio de 2005) en 19 ciudades colombianas. Se diseñó una base de datos de los registros de consumo de medicamentos de la empresa dispensadora de fármacos. Los datos se analizaron con el paquete estadístico SPSS 13 para Windows, empleando la prueba de la $\mathrm{t}$ de Student y el análisis de la varianza (ANOVA) para comparar variables cuantitativas y la prueba de la $\chi^{2}$ para comparar variables categóricas.

Resultados. La edad media de los pacientes era de 60,7 $\pm 12,7$ años; el 56,6 \% eran mujeres, de edad media significativamente mayor que la de los hombres $(\mathrm{P}<0,05)$. El 48\% de las personas recibía monoterapia y el $52 \%$, politerapia con entre dos y cuatro antidiabéticos. Los antidiabéticos prescritos fueron: biguanidas (67,5\% de los casos), sulfonilureas (64,9\%), insulinas $(23,5 \%)$ y tiazolidindionas $(0,1 \%)$. Las asociaciones más empleadas fueron: glibenclamida + metformina $(n=2847)$, metformina + insulina $(n=510)$, glibenclamida + insulina $(n=148)$ y metformina + insulina + glibenclamida $(n=288)$. El $94,3 \%$ de los pacientes recibían un tratamiento concomitante: antihipertensivos (74,4\%), antiinflamatorios $(61,5 \%)$, hipolipemiantes $(45,5 \%)$, antiulcerosos $(21 \%)$, psicofármacos $(16,8 \%)$, antiinfecciosos $(14,4 \%)$, antiasmáticos $(5,3 \%)$ y ácido acetilsalicílico $(2,8 \%)$. La comedicación era más frecuente en las mujeres que en los hombres (95,6\% frente a 92,7\%, P < 0,001). Probablemente haya subempleo de algunos fármacos (metformina, tiazolodindionas, inhibidores de la enzima convertidora de la angiotensina [IECA] y ácido acetilsalicílico) y sobreempleo de otros (antiulcerosos).

Conclusiones. Existen diferencias significativas en las formulaciones de médicos en las 19 ciudades estudiadas, pero predominan patrones de prescripción racionales. Se plantea así la necesidad de diseñar estrategias educativas para corregir algunos hábitos de prescripción y explorar los resultados clínicos de las prescripciones estudiadas.

Palabras clave Diabetes mellitus, agentes antidiabéticos, prescripción de medicamentos, Colombia.

1 Universidad Tecnológica de Pereira, Facultad de Ciencias de la Salud, Pereira, Colombia. La correspondencia deberá dirigirse a Jorge Enrique Machado, Audifarma S.A., calle 105 N. ${ }^{\circ} 14-140$, Pe- reira, Risaralda, Colombia; tel.: (57 6) 3137800 ext. 119, fax: (57 6) 3137822, correo electrónico: jormach@telesat.com.co.

2 Audifarma S.A., Pereira, Risaralda, Colombia.
La diabetes mellitus es una enfermedad que se está propagando en todo el mundo y se ha convertido en un serio 
problema de salud pública (1-3). La diabetes mellitus de tipo 2 corresponde al 90-95\% de los casos $(1,2,4)$. Se estima que la diabetes mellitus afecta a aproximadamente 130 millones de personas en el mundo, de las cuales, 35 millones viven en el continente americano y entre 15 y 19 millones, en América Latina. Se prevé que en el año 2025 habrá aproximadamente 300 millones de diabéticos en el mundo y 40 millones en América Latina $(1,2,5,6)$. La prevalencia mundial de la diabetes mellitus se halla entre el $5 \%$ y el $8 \%(2,3,5-8)$; en Colombia, las cifras son similares: entre el 5,1\% y el $7,0 \%$ de los adultos $(1,9,10)$.

La diabetes mellitus se asocia a una mayor probabilidad de muerte prematura, debido principalmente al elevado riesgo de enfermedades cardiovasculares y metabólicas $(1,2)$-en particular, hipertensión arterial y dislipidemia $(11,12)$ - al que se suma un mayor riesgo de ceguera, insuficiencia renal, neuropatía y amputación de miembros inferiores, lo que produce una disminución importante de la calidad de vida de quienes la padecen (13) y una enorme carga económica para la sociedad $(3,14)$. Sin embargo, los costos pueden reducirse de manera significativa mediante un control adecuado de la glucemia y de los factores de riesgo cardiovascular asociados, una buena educación y un correcto programa de reconocimiento temprano de las complicaciones $(6,14)$.

El tratamiento del paciente con diabetes mellitus abarca medidas no farmacológicas que incluyen el seguimiento de un plan de alimentación personalizado que garantice el aporte calórico adecuado para mantener el peso ideal (índice de masa corporal [IMC] comprendido entre 19 y 25), la corrección de comorbilidades asociadas - tales como la hipercolesterolemia, la hipertrigliceridemia, la hipertensión arterial y la insuficiencia renal-, la realización de un plan de ejercicios adecuado y la adopción de comportamientos saludables, como no fumar $(6,15)$.

Además de estas medidas, los pacientes diabéticos pueden necesitar medidas farmacológicas. Los medica- mentos antidiabéticos más importantes son: fármacos que estimulan la secreción de insulina (sulfonilureas - como la glibenclamida-, nateglinida y otros secretagogos rápidos), reductores de la producción hepática de glucosa (biguanidas, como la metformina), retardadores de la absorción y la digestión intestinal de carbohidratos (inhibidores de la --glucosidasa), mejoradores de la acción de la insulina (tiazolidindionas, biguanidas) $(6,16-21)$ e insulinas.

El tratamiento general del paciente con diabetes mellitus debe incluir estas dos clases de medidas, las cuales reducen las complicaciones agudas y, a largo plazo, las complicaciones microvasculares y macrovasculares. Se estima que por cada un $1 \%$ de disminución de la concentración de glucohemoglobina se reducen en un 30\% las complicaciones microvasculares (13).

La metformina se ha convertido en uno de los medicamentos más importantes para el tratamiento de los pacientes con diabetes mellitus de tipo 2 . Se trata actualmente de la principal opción terapéutica en caso de sobrepeso u obesidad $(16,17,20-22)$ porque tiene efectos beneficiosos sobre el peso (16, $23)$, la resistencia a la insulina $(22,23)$ y las concentraciones séricas de lípidos $(24,25)$, reduce la inflamación vascular y mejora la función endotelial.

Diferentes estudios plantean que en los obesos la resistencia a la insulina es una característica predominante y que estos pacientes se ven más beneficiados con medicamentos que favorecen la sensibilidad a la insulina, como la metformina o las tiazolidindionas, mientras que los pacientes con peso normal responden a fármacos que estimulan la liberación de insulina $(13,18)$. Incluso se puede emplear politerapia con diferentes agentes farmacológicos (6).

Uno de los grandes retos que debe afrontar el Sistema General de Seguridad Social en Salud (SGSSS) y los organismos de salud colombianos es el mejoramiento en la detección, el acceso al tratamiento y el incremento de las tasas de control de los pacientes diabéticos. En 1995 se creó en Colombia el Instituto Nacional para la Vigilancia de Me- dicamentos y Alimentos (INVIMA) y, dentro de un marco de uso adecuado de productos farmacéuticos, se adoptó un listado de medicamentos esenciales del Plan Obligatorio de Salud (POS), en el que se incluyen los antidiabéticos que pueden emplearse en el programa de control de la diabetes de las instituciones de salud del SGSSS: insulinas (insulina cristalina e insulina NPH -Neutral Protamine Hagedorn-), sulfonilureas (glibenclamida) y biguanidas (metformina) $(26,27)$.

El objetivo de este estudio fue determinar los patrones de prescripción de medicamentos antidiabéticos en una población de personas afiliadas al SGSSS, definiendo la frecuencia de prescripción de cada uno de los antidiabéticos empleados con mayor regularidad, describiendo la comedicación empleada relacionada con enfermedades de importancia clínica para el diabético e identificando las principales variables sociodemográficas asociadas a la prescripción de antidiabéticos, con el fin de mejorar los conocimientos acerca del tratamiento farmacológico de la diabetes mellitus, para favorecer el uso racional de medicamentos en el contexto de la salud pública.

\section{MATERIALES Y MÉTODOS}

Se realizó un estudio descriptivo observacional sobre la utilización de medicamentos en una población de 1836777 personas: 1734726 afiliadas al régimen contributivo del SGSSS en cuatro Empresas Promotoras de Salud (EPS) y 102051 afiliadas a una Administradora de Régimen Subsidiado (ARS). Se incluyeron en el estudio los 7308 pacientes con diagnóstico de diabetes mellitus de tipo 1 ó 2, de todas las edades y de ambos sexos, que habían sido atendidos en el programa de atención médica de control de la diabetes mellitus durante el mes de julio de 2005 y habían recibido tratamiento antidiabético durante por lo menos los tres meses anteriores, en 19 ciudades colombianas donde se dispone de bases de datos pertinentes y confiables: Arbelaez, Armenia, Bucaramanga, Bogotá, Barranquilla, Buga, 
Cali, Cartagena, Fusagasuga, Ibagué, Manizales, Medellín, Pereira, San Bernardo, San José del Guaviare, Santa Marta, Valledupar, Villavicencio y Villeta.

El requisito de tratamiento antidiabético de por lo menos tres meses de duración se fijó para garantizar que los pacientes presentaban adhesión al tratamiento de manera más o menos estable y tolerabilidad a la medicación, y poder suponer cierto grado de control glucémico. De esta manera, se excluyeron las personas con diagnóstico reciente de diabetes mellitus y las que no asistieron a las citas médicas al final del período de observación de tres meses, por considerarse que estos individuos podían introducir sesgos en la evaluación de los patrones de empleo de medicamentos utilizados de manera continua y prolongada.

A partir de la información sobre los consumos de medicamentos, capturada de manera sistemática e ininterrumpida por la empresa que dispensaba los medicamentos a la población afiliada, con la colaboración del Departamento de Sistemas de dicha empresa, se diseñó una base de datos que permitió recoger los siguientes grupos de variables de los pacientes que recibieron medicamentos antidiabéticos en el período de observación (28):

1. Variables demográficas: edad, sexo, tipo de afiliación al SGSSS.

2. Medicamentos antidiabéticos dispensados, con sus dosis respectivas: insulinas (cristalina, NPH, lispro, glargina, etc.), secretagogos (sulfonilureas - como la glibenclamida, la gliclazida y la tolbutamida- y meglitinidas — como la nateglinida y la repaglinida-), medicamentos que mejoran la sensibilidad periférica a la insulina (biguanidas - como la metformina - y tiazolidinedionas - como la rosiglitazona y la pioglitazona-) y retardadores de la absorción de carbohidratos (inhibidores de la $\alpha$-glucosidasa, como la acarbosa)

3. Comedicación dispensada: a) antihipertensivos (inhibidores de la enzima convertidora de la angiotensina [IECA], diuréticos, betablo- queadores, calcio, metildopa y prazosin); b) antiagregantes plaquetarios (ácido acetilsalicílico); c) antiinflamatorios (ibuprofeno, naproxeno, diclofenaco, corticosteorides); d) antiarrítmicos (amiodarona, propafenona); e) inotrópicos (digoxina, metildigoxina); f) hipolipemiantes (lovastatina, gemfibrozil); g) antiulcerosos (omeprazol, ranitidina, sucralfato, antiácidos); h) antiasmáticos inhalatorios (beclometasona, salbutamol); i) estrógenos y calcio; j) antianginosos (isosorbide); k) hormona tiroidea.

En los siguientes casos se consideró la comedicación como un indicador sustituto de enfermedad crónica asociada con la diabetes: antihipertensivos (hipertensión arterial), digitálicos (insuficiencia cardíaca o fibrilación auricular), hipolipemiantes (dislipidemia), amiodarona o propafenona (arritmias), nitratos (enfermedad coronaria), broncodilatadores o beclometasona (asma o enfermedad pulmonar obstructiva crónica [EPOC]). En estos casos, se analizó la conveniencia o no del tipo de antidiabético elegido, según el tipo de comorbilidad. Por último, se revisaron las combinaciones de medicamentos que podían ocasionar interacciones potencialmente riesgosas con el propósito de recomendar medidas correctivas.

El protocolo del estudio se sometió a la aprobación del Comité de Bioética de la Facultad de Ciencias de la Salud de la Universidad Tecnológica de Pereira y de los Comités de Ética de las EPS, en la categoría de "investigación sin riesgo", según la resolución $\mathrm{N}^{\circ}$ 8430 de 1993 del Ministerio de Salud de Colombia, que establece las normas científicas, técnicas y administrativas para la investigación en salud.

La base de datos fue revisada por el Departamento de Farmacoepidemiología de la empresa encargada de la dispensación farmacéutica y validada mediante una prueba piloto con los pacientes de Pereira. Para el análisis de los datos, se utilizó el paquete estadístico SPSS 13 para Windows. Se emplearon las pruebas de la $t$ de Student y el análisis de la varianza (ANOVA) para comparar variables cuantitativas y la prueba de la $\chi^{2}$ para comparar variables categóricas. Se aplicaron modelos de regresión logística binaria usando como variables dependientes el tipo de tratamiento antidiabético (monoterapia/politerapia antidiabética) y el empleo de comedicación (sí/no), y como covariables las asociadas significativamente con las variables dependientes en los análisis con dos variables. El nivel de significación estadística se fijó en $0,05(P<0,05)$.

\section{RESULTADOS}

De las 7308 personas que habían recibido tratamiento antidiabético durante el período mínimo de tres meses, $4134(56,6 \%)$ eran mujeres y 3174 $(43,4 \%)$, hombres. La edad media era de 60,7 \pm 12,7 años (rango: 0-96 años), con una diferencia significativa entre los hombres y las mujeres $(59,7$ $\pm 12,7$ años frente a $61,4 \pm 12,6$ años; $P<0,001)$. La figura 1 muestra la distribución por edad y por sexo de este grupo de pacientes.

En el cuadro 1 se resumen los patrones de prescripción de los cuatro antidiabéticos utilizados: los tres fármacos incluidos en el listado de medicamentos esenciales, con los que se lleva a cabo el programa de control de la diabetes en Colombia, y la rosiglitazona, prescrita a seis pacientes del estudio. Considerándolos por grupos farmacológicos los agentes más utilizados fueron, en orden decreciente: las biguanidas, las sulfonilureas y las insulinas.

\section{Monoterapia y politerapia}

De los 7308 pacientes diabéticos incluidos en el estudio, 3509 (48,0\%) eran tratados con un solo antidiabético y 3799 (52,0\%) con una asociación de dos o más. En la figura 2 se ilustra la relación monoterapia/politerapia para cada uno de los cuatro antidiabéticos evaluados; cabe destacar que en todos los casos fue más frecuente el empleo en politerapia que en monoterapia. Las asociaciones utilizadas fueron, en orden decreciente de frecuencia: gli- 
FIGURA 1. Distribución de los 7308 pacientes diabéticos incluidos en el estudio según la edad y el sexo, Colombia, 2005

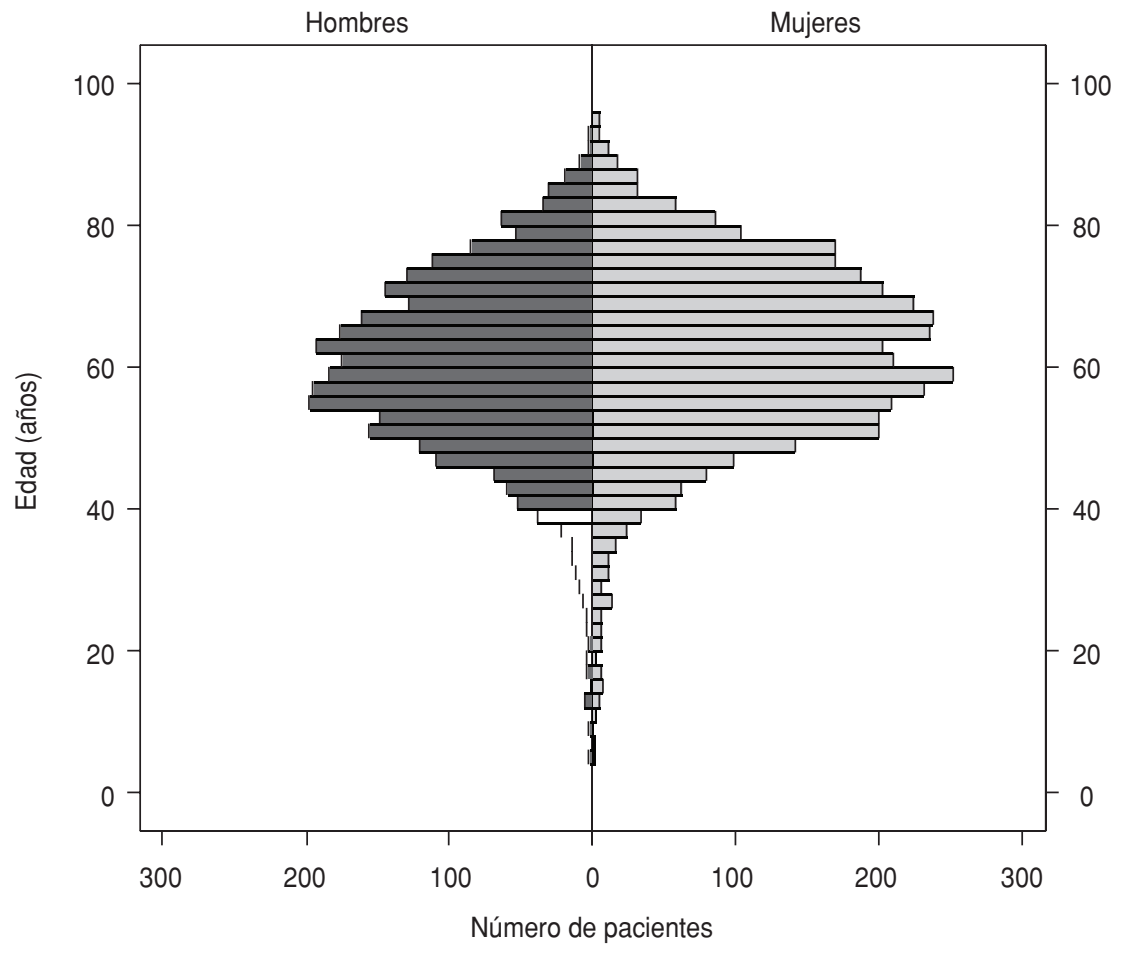

benclamida + metformina $(n=2847$, $39,0 \%$ de los individuos tratados con politerapia), metformina + insulina $(\mathrm{n}=510,7,0 \%)$, glibenclamida + insulina $(\mathrm{n}=148,2,0 \%)$, metformina + insulina + glibenclamida $(\mathrm{n}=288$, $3,9 \%)$, insulina + rosiglitazona $(\mathrm{n}=2)$, metformina + glibenclamida + rosiglitazona $(\mathrm{n}=2)$, insulina + glibenclamida + rosiglitazona $(n=1)$ y metformina + glibenclamida + insulina + rosiglitazona $(n=1)$.
Según el análisis de regresión logística binaria, el tratamiento con monoterapia antidiabética se asocia significativamente con las covariables "grupo de edad" y "ciudad". En efecto, las personas del grupo de adultos maduros (entre 45 y 60 años) tienen una probabilidad 1,3 veces mayor de recibir un tratamiento con un solo antidiabético que los pacientes de edades superiores (riesgo relativo [RR]: 1,345; intervalo de confianza del 95\% [IC95\%]: 1,103-
1,639; $P=0,003)$. Asimismo en Bogotá (RR: 1,210; IC95\%: 1,001-1,462; $P=$ 0,048), Ibagué (RR: 1,343; IC95\%: 1,0481,722; $P=0,02$ ) y Medellín (RR: 1,378; IC95\%: 1,092-1,740; $P=0,007)$ el tratamiento con monoterapia antidiabética es más probable que en las otras ciudades del estudio.

\section{Comedicación}

La diabetes mellitus es un síndrome que se acompaña frecuentemente de comorbilidad o daño en órganos blanco, lo que necesita un tratamiento adicional. Entre los diabéticos incluidos en este estudio, 6893 (94,3\%) recibían un tratamiento concomitante con uno o varios de los siguientes grupos de medicamentos que reflejan comorbilidad o pueden tener interacciones con algunos antidiabéticos: antihipertensivos (5 435, 74,4\%), antiinflamatorios $(4497,61,5 \%)$, hipolipemiantes (3 322, 45,5\%), antiulcerosos (1 532, $21 \%)$, psicofármacos (1 225, 16,8\%), antiasmáticos (386, 5,3\%), ácido acetilsalicílico en dosis antiagregantes plaquetarias (201, 2,8\%), antianginosos (177, 2,4\%), inótrópicos $(124,1,7 \%)$ y antiarrítmicos (55, 0,8\%).

Al analizar mediante regresión logística binaria la relación entre el empleo de comedicación y otras variables se encuentra una asociación significativa con las covariables "grupo de edad", "sexo" y "ciudad". La comedicación era más frecuente en las mujeres que en los hombres $(95,6 \%$ frente a 92,7\%; RR: 1,635; IC95\%: 1,332-2,008;

CUADRO 1. Patrones de prescripción de los cuatro antidiabéticos utilizados en el tratamiento de un grupo de 7308 pacientes diabéticos, Colombia, 2005

\begin{tabular}{|c|c|c|c|c|c|c|c|c|}
\hline \multirow[b]{2}{*}{ Antidiabético } & \multicolumn{4}{|c|}{ Pacientes tratados } & \multicolumn{3}{|c|}{ Dosis diaria prescrita } & \multirow[b]{2}{*}{$\begin{array}{c}\text { Relación } \\
\text { politerapia/ } \\
\text { monoterapia }\end{array}$} \\
\hline & \begin{tabular}{l}
\multicolumn{2}{c}{ Total } \\
No. $(\%)$
\end{tabular} & $\begin{array}{c}\text { Edad } \\
\text { Media } \pm \text { DE } \\
\text { (años) }\end{array}$ & $\begin{array}{l}\text { Mujeres } \\
\text { No. (\%) }\end{array}$ & $\begin{array}{l}\text { Hombres } \\
\text { No. (\%) }\end{array}$ & Media & Moda & Rango & \\
\hline Metformina ${ }^{a}$ & $4931(67,5)$ & $56,7 \pm 18,5$ & $2793(56,6)$ & $2138(43,4)$ & $1624,2 \mathrm{mg}$ & $1700 \mathrm{mg}$ & $850,0-5950 \mathrm{mg}$ & $2,8 / 1,0$ \\
\hline Glibenclamida $^{a}$ & $4743(64,9)$ & $57,5 \pm 18,3$ & $2657(56,0)$ & $2086(44,0)$ & $10,7 \mathrm{mg}$ & $10 \mathrm{mg}$ & $1,0-60 \mathrm{mg}$ & $2,3 / 1,0$ \\
\hline Insulina ${ }^{a}$ & $1718(23,5)$ & $53,8 \pm 20,7$ & $1001(58,3)$ & $717(41,7)$ & 63,6 UI & 33,3 U & 33,3-1 $000 \mathrm{UI}$ & $1,2 / 1,0$ \\
\hline Rosiglitazona & $6(0,1)$ & $62,6 \pm 8,8$ & $1(16,7)$ & $5(83,3)$ & $7,2 \mathrm{mg}$ & $4,0 \mathrm{mg}$ & $3,7-12 \mathrm{mg}$ & $6,0 / 0,0$ \\
\hline
\end{tabular}

\footnotetext{
a Antidiabéticos incluidos en el listado colombiano de medicamentos esenciales.
} 
FIGURA 2. Frecuencia de prescripción de los tres antidiabéticos incluidos en el listado de medicamentos esenciales, en monoterapia o politerapia, Colombia, 2005

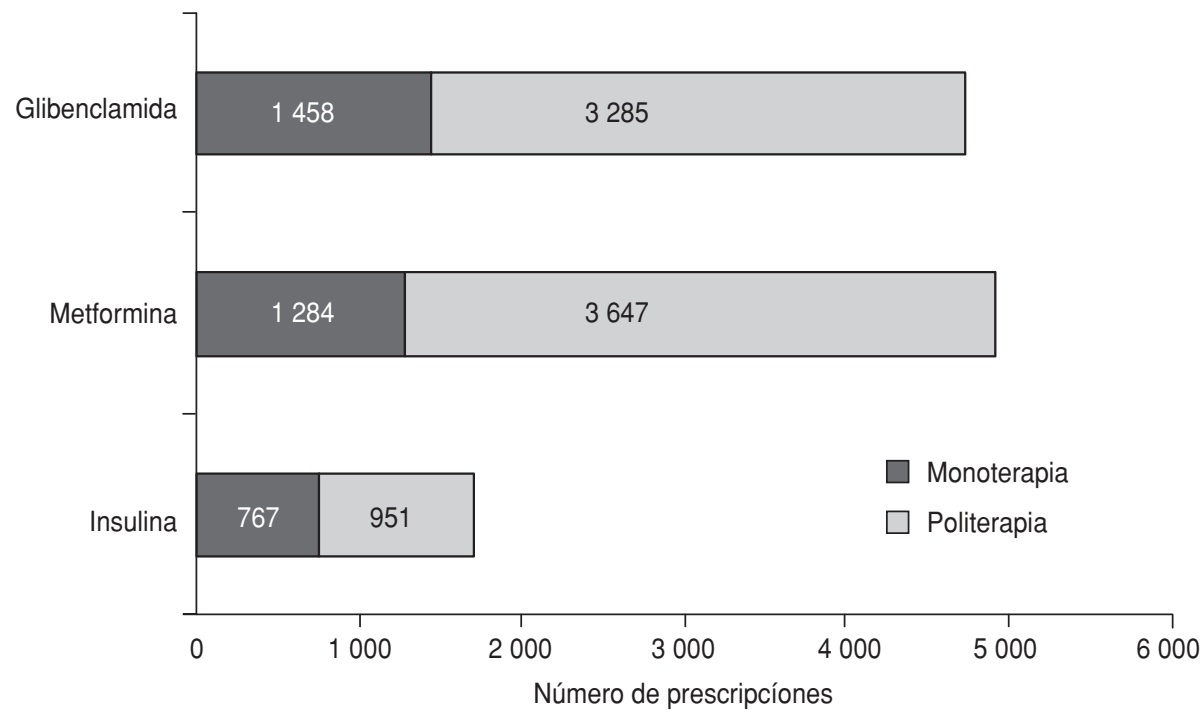

$P<0,001)$ y en los mayores de 18 años que en los menores $(93,8 \%$ frente a 80,7\%; RR: 0,589; IC95\%: 0,416-0,833; $P=0,003)$. Al comparar los porcentajes de comedicación por ciudad, se observan valores significativamente menores en Cali (RR: 1,537; IC95\%: 1,0082,343; $P=0,04)$ y en Cartagena (RR: 1,755; IC95\%: 1,044-2,949; $P=0,03$ ) y significativamente mayores en Manizales (RR: 0,351; IC95\%: 0,183-0,673; $P=0,002$ ) y Medellín (RR: 0,405; IC95\%: 0,217-0,757; $P=0,005)$ con respecto a la ciudad de Pereira, tomada como modelo (RR: 1,106; IC95\%: $0,672-1,820 ; P=0,691$ ) (cuadro 2).

Cuando se relaciona la comedicación con el medicamento prescrito para el tratamiento concomitante de la hipertensión arterial, se observa que dos enfermedades era tratado con IECA.

\section{Combinaciones de fármacos}

Al estudiar las combinaciones de medicamentos prescritos se encontraron algunos que no conllevan el riesgo de interacciones potencialmente peligrosas, pero requieren especial cuidado porque pueden interferir con el adecuado control metabólico de la diabetes: diuréticos $(2337,31,9 \%$ de los diabéticos), agonistas $\beta_{2}$ (204, $2,8 \%)$, prednisolona $(128,1,75 \%)$, estrógenos + progestágenos $(42,0,57 \%)$ sólo el $75,4 \%$ de los pacientes con las -que pueden elevar la glucemia-y propanolol $(67,0,91 \%)$-que puede enmascarar la hipoglucemia-. Entre los pacientes tratados con glibenclamida, se encontraron 54 casos de prescripción concomitante de trimetoprim con sulfametoxazol, que puede provocar hipoglucemia.

\section{Comparación entre ciudades}

Se compararon variables demográficas y algunos indicadores de prescripción entre las 19 ciudades colombianas incluidas en este estudio. Sin embargo, debido al reducido número de pacientes en algunas de ellas, en los cuadros 2 y 3 solo se incluyeron las ocho ciudades mayores, que abarcan el 92,5\% de los pacientes incluidos, y se hizo el mismo análisis con los datos globales del estudio. Los consumos de los cuatro antidiabéticos del estudio se presentan como relación entre la dosis media administrada en cada ciudad y la dosis diaria definida (DDD) y como número de dosis diarias definidas por 1000 habitantes (cuadro 3), para que puedan ser útiles en comparaciones posteriores.

\section{DISCUSIÓN}

El presente estudio permitió determinar en un grupo de pacientes afiliados al SGSSS en Colombia los patrones de prescripción de antidiabéticos, la comedicación más frecuente y otras variables asociadas. Esta información puede ser utilizada por los administra-

CUADRO 2. Comparación de variables demográficas y de indicadores de prescripción de antidiabéticos entre ocho ciudades incluidas en el estudio, Colombia, 2005

\begin{tabular}{|c|c|c|c|c|c|c|c|c|c|}
\hline & $\begin{array}{c}\text { Bogotá } \\
n=2039\end{array}$ & $\begin{array}{c}\text { Barranquilla } \\
n=1009\end{array}$ & $\begin{array}{c}\text { Cali } \\
n=1101\end{array}$ & $\begin{array}{c}\text { Cartagena } \\
n=363\end{array}$ & $\begin{array}{l}\text { Ibagué } \\
n=470\end{array}$ & $\begin{array}{c}\text { Manizales } \\
n=562\end{array}$ & $\begin{array}{c}\text { Medellín } \\
n=610\end{array}$ & $\begin{array}{l}\text { Pereira } \\
n=603\end{array}$ & $\begin{array}{c}\text { Global } \\
n=7308\end{array}$ \\
\hline Edad media (años) & 61,9 & 60,4 & 59,7 & 61,6 & 61,3 & 60,6 & 59,4 & 59,2 & 60,7 \\
\hline Monoterapia (\%) & 46,7 & 52,9 & 48,3 & 49,0 & 43,6 & 49,5 & 43,1 & 46,9 & 48,0 \\
\hline Comedicación (\%) & 95,5 & 93,6 & 90,7 & 90,9 & 95,1 & 97,5 & 97,4 & 93,5 & 94,3 \\
\hline \multicolumn{10}{|c|}{ Tasas de prescripción (\%) } \\
\hline Metformina & 71,8 & 53,7 & 67,8 & 65,8 & 73,2 & 60,1 & 73,1 & 72,5 & 67,5 \\
\hline
\end{tabular}


CUADRO 3. Comparación de indicadores de consumo de antidiabéticos (relación entre la dosis media administrada y la dosis diaria definida [DDD] y número de DDD por 1000 habitantes por día) entre ocho ciudades incluidas en el estudio, Colombia, 2005

\begin{tabular}{|c|c|c|c|c|c|c|c|c|c|}
\hline & $\begin{array}{c}\text { Bogotá } \\
(n=2039)\end{array}$ & $\begin{array}{l}\text { Barranquilla } \\
(n=1009)\end{array}$ & $\begin{array}{c}\text { Cali } \\
(n=1101)\end{array}$ & $\begin{array}{l}\text { Cartagena } \\
(n=363)\end{array}$ & $\begin{array}{l}\text { Ibagué } \\
(n=470)\end{array}$ & $\begin{array}{c}\text { Manizales } \\
(n=562)\end{array}$ & $\begin{array}{l}\text { Medellín } \\
(n=610)\end{array}$ & $\begin{array}{l}\text { Pereira } \\
(n=603)\end{array}$ & $\begin{array}{c}\text { Global } \\
(n=7308)\end{array}$ \\
\hline \multicolumn{10}{|c|}{$\begin{array}{l}\text { Relación entre la dosis media } \\
\text { diaria administrada y la DDDa }\end{array}$} \\
\hline Metformina & 0,8 & 0,7 & 0,8 & 0,7 & 0,9 & 0,7 & 0,9 & 0,8 & 0,8 \\
\hline Glibenclamida & 1,2 & 1,1 & 1,0 & 1,0 & 1,1 & 1,1 & 1,2 & 1,0 & 1,1 \\
\hline Insulina & 1,6 & 1,7 & 1,9 & 1,7 & 1,5 & 1,3 & 1,5 & 1,3 & 1,6 \\
\hline \multicolumn{10}{|c|}{ Número de DDDa/ } \\
\hline Glibenclamida & 0,7 & 1,0 & 1,2 & 0,9 & 1,6 & 1,6 & 1,1 & 1,0 & 1,0 \\
\hline Insulina & 1,7 & 2,0 & 3,9 & 2,1 & 2,8 & 2,8 & 2,1 & 1,4 & 2,2 \\
\hline
\end{tabular}

a DDD de metformina: $2 \mathrm{~g}$; DDD de glibenclamida: $10 \mathrm{mg}$; DDD de insulina: $60 \mathrm{UI}$.

dores de salud para tomar decisiones dirigidas a mejorar la atención sanitaria de los pacientes diabéticos.

La edad media y la relación de sexos del grupo de pacientes incluidos en esta investigación fueron similares a las notificadas en otros estudios (29-31).

Los tres principales antidiabéticos analizados en este estudio -metformina, glibenclamida e insulina - figuran en el listado colombiano de medicamentos esenciales y como tales poseen elevado valor intrínseco. Por consiguiente, estos fármacos no sólo se consideran suficiente, sino de primera elección para el tratamiento de la diabetes (26). Tratándose de un listado cerrado de medicamentos, se entiende por qué el 99,9\% de los pacientes del estudio son tratados con estos tres fármacos.

La tasa de prescripción de metformina a los pacientes con diabetes de tipo $2(67,5 \%)$ puede considerarse adecuada y sobrepasa la cifra notificada para América Latina, de sólo el 14\% (14). Las 767 personas tratadas exclusivamente con insulina $(10,5 \%)$ son probablemente los pacientes con diabetes mellitus de tipo 1 del estudio; esta proporción coincide con la notificada en los informes epidemiológicos sobre la enfermedad (10). Puede considerarse que la monoterapia con metformina $(17,6 \%$ de los casos) y con insulina es adecuada.

En el caso de la glibenclamida, su elevada utilización en monoterapia
(20\%) no parece justificarse, excepto en los pacientes con diabetes de tipo 2 e índice de masa corporal menor de 27, pero este dato se desconoce y escapa de los alcances del presente estudio $(6,15)$. En la mayoría de los casos, debería considerarse la utilización de medicamentos que mejoren la sensibilidad periférica a la insulina.

Las dosis prescritas de los antidiabéticos analizados se encuentran dentro de los intervalos recomendados por consenso internacional (6), pero la dosis diaria administrada de metformina, que en promedio fue del $81 \%$ de la recomendada, es claramente es inferior a la dosis diaria definida, lo que puede interpretarse de dos maneras: nuestros pacientes se controlan con dosis menores del medicamento o están siendo tratados con dosis subterapéuticas. En América Latina se encontró una proporción importante de pacientes insuficientemente tratados, con dosis subterapéuticas de insulina o de biguanidas, y ausencia de tratamiento específico en casos de comorbilidad con hipertensión y dislipidemia (14).

La hipertensión arterial y la dislipidemia fueron las comorbilidades más frecuentes en este estudio (74,4 y $45,5 \%$, respectivamente). El 61,5\% de los diabéticos recibían un tratamiento concomitante con antiinflamatorios no esteroides (AINE), lo cual puede indicar una alta prevalencia de enfermedades inflamatorias o un uso inadecuado de estos medicamentos. Los AINE dis- minuyen la eficacia del tratamiento antihipertensivo y, por su acción sobre prostaglandinas renales, se asocian con daño renal (32). Hay que tener presente que los hipertensos y los diabéticos, aun estando controlados, pueden llegar a padecer daño renal y, por lo tanto, antes de prescribir un tratamiento con AINE a estos pacientes es preciso evaluar cuidadosamente la relación riesgo/beneficio.

Sólo un pequeño grupo de pacientes recibía ácido acetilsalicílico en dosis antiagregantes plaquetarias, a pesar de las recomendaciones basadas en resultados obtenidos en estudios científicos (33-35). Además, el 24,6\% de los diabéticos con hipertensión no recibían IECA, en un aparente desconocimiento de los beneficios de tales fármacos en estas circunstancias clínicas (28).

Las diferencias en los patrones de prescripción entre las diferentes ciudades colombianas del estudio (cuadros 2 y 3) no son sorprendentes, ya que la variabilidad en la atención médica, en particular en los hábitos de prescripción, es un hallazgo constante en los estudios farmacoepidemiológicos. Estas variaciones, de importancia por sus implicaciones médicas, sociales, económicas y de salud pública, están relacionadas con características de los pacientes (edad, sexo, comorbilidad) y con estilos de práctica profesional que también resultan de factores personales y de la formación y del entrenamiento de los prescriptores (36). 
Las limitaciones para la interpretación de algunos resultados de esta investigación pueden superarse con otros estudios de utilización de medicamentos, correspondientes a la segunda fase de esta línea de investigación, donde se cuente con información acerca de la clasificación según el tipo de diabetes mellitus, de los intervalos de dosis empleados, de la incidencia de reacciones adversas atribuibles a la medicación, de la morbilidad asociada, de la adhesión al tratamiento y del grado de control de la diabetes.

Basándose en los patrones de prescripción encontrados en el presente estudio puede afirmarse que, en general, predominan los hábitos de prescripción racional, con el empleo de medicamentos de alto valor terapéutico, en dosis usuales y en politerapia, con suficiente respaldo científico. Sin embargo, en el caso de las biguanidas, se puede estar frente a un subempleo y a una utilización de dosis inferiores a las definidas internacionalmente. El elevado uso de sulfonilureas plantea la posibilidad de que no se haya reconocido en algunos diabéticos el trastorno en la sensibilidad periférica a la insulina y que se siga tratándolos como insulinodeficientes.

El subempleo de algunos fármacos detectado en este estudio, en particular de IECA en diabéticos hipertensos y de ácido acetilsalicílico en dosis antiagregantes plaquetarias, muestra que un porcentaje importante de pacientes no se beneficia de los efectos científicamente demostrados de estos medicamentos. Los resultados de este trabajo pueden constituir herramientas útiles para los administradores de sistemas y servicios de salud, que les permitan tomar decisiones frente a la forma en que sus médicos tratan este grupo de pacientes, para mejorar algunos patrones de uso y reducir así las complicaciones agudas y a largo plazo.

Es importante que los médicos tratantes en las diferentes ciudades colombianas conozcan los resultados de este estudio para conseguir que aumenten la prescripción de metformina y revisen la efectividad de este fármaco en las dosis en que está siendo administrado, así como lograr que aumente el porcentaje de diabéticos hipertensos tratados con IECA o con antagonistas de los receptores de la angiotensina II (ARA II), y que todos los pacientes reciban un tratamiento con ácido acetilsalicílico para prevenir el riesgo cardiovascular.

\section{REFERENCIAS}

1. Pan American Health Organization. Diabetes in the Americas. Epidemiol Bull. 2001; 22(2): 1-3.

2. Brower V. Like a snake in the grass. As the incidence of type 2 diabetes escalates, new developments offer hope for better treatments, EMBO Rep. 2004;5:555-8.

3. Chiasson JL, Rabasa-Lhoret R. Prevention of type 2 diabetes. Insulin resistance and B-cell function. Diabetes. 2004;53:s34-8.

4. Montoya Y, Grisales H, Sepulveda H. Mortalidad por diabetes mellitus, Medellín, 19871988. Rev Fac Nac Salud Pública Univ Antioquia. 2004;22:47-59.

5. Curtis J, Wilson C. Preventing type 2 diabetes mellitus. J Am Board Fam Pract 2005;18:37-43.

6. Asociación Latinoamericana de Diabetes. Guías ALAD de diagnóstico, control y tratamiento de la diabetes mellitus tipo 2. Rev Asoc Latinoam Diabetes. 2000;1:101-66.

7. Clemens A, Riemann JF, Siegel EG. Optimized diabetes therapy in type 2 diabetics. Med Klin (Munich). 2003;98:484-92.

8. Barceló A, Daroca M, Ribera R, Duarte E, Zapata A, Vohra M. Diabetes in Bolivia. Rev Panam Salud Pública. 2001;10:318-23.

9. Aschner P, King H, Triana de Torrado M, Rodríguez BM. Glucose intolerance in Colombia. A population-based survey in an urban community. Diabetes Care. 1993;16:90-3.

10. Aschner P. Diabetes trends in Latin America. Diabetes Metab Res Rev. 2002;18:s27-31.

11. Skyler JS, Oddo C. Diabetes trends in the USA. Diabetes Metab Res Rev. 2002;18:s21-6.
12. Fonseca VA. Management of diabetes mellitus and insulin resistance in patients with cardiovascular disease. Am J Cardiol. 2003;92: 50j-60j.

13. Clarke PM, Gray AM, Briggs A, Stevens RJ, Matthews DR, Holman RR. Cost-utility analyses of intensive blood glucose and tight blood pressure control in type 2 diabetes (UKPDS 72). Diabetologia. 2005;48:868-77.

14. Gagliardino JJ, de la Hera M, Siri F y Grupo de Investigación de la Red QUALIDIAB. Evaluación de la calidad de la asistencia al paciente diabético en América Latina. Rev Panam Salud Pública. 2001;10:309-17.

15. Centers for Disease Control and Prevention. Prevalence of Overweight and Obesity Among Adults with Diagnosed Diabetes, United States, 1988-1994 and 1999-2002. MMWR. 2004;53:1066-8.

16. Krentz AJ, Bailey CJ. Oral antidiabetic agents: current role in type 2 diabetes mellitus. Drugs. 2005;65:385-411.

17. Hamman A, Morcos M, Nawroth P. Oral diabetes treatment. Which substance is indicated at which time? Internist (Berl). 2004;45: 1356-63.

18. Rendell M. The role of sulphonylureas in the treatment of type 2 diabetes mellitus. Drugs. 2004;64:1339-58.

19. Bell DS. Type 2 diabetes mellitus: what is the optimal treatment regimen? Am J Med. 2004; $116: 23 \mathrm{~s}-29 \mathrm{~s}$

20. Malik T, Trence DL. Treatment diabetes using oral agents. Prim Care. 2003;30:527-41.
21. Lebovitz H. Oral antidiabetic agents: 2004. Med Clin North Am. 2004;88:847-63.

22. Tankova T. Current indications for metformin therapy. Rom J Intern Med. 2003;41:215-25.

23. Kirpichnikov D, McFarlane S, Sowers J. Metformin: An update. Ann Intern Med. 2002;137: 25-33.

24. Henry RR. Insulin Resistance: from predisposing factor to therapeutic target in type 2 diabetes. Clin Ther. 2003;25:b47-63.

25. Zangeneh F, Kudva YC, Basu A. Insulin sensitizers. Mayo Clin Proc. 2003;78:471-9.

26. Colombia, Ministerio de Salud. Consejo Nacional de Seguridad Social en Salud. Acuerdo 228 de 2002. Diario Oficial. Hallado en: http:/ / www.minproteccionsocial.gov.co/ VBeContent/NewsDetail.asp?ID=14774\&IDCompany=3 Acceso el 31 mayo de 2006.

27. Colombia, Ministerio de Salud. Resolución 5061 de 1997. Diario Oficial. Hallado en: http://www.minproteccionsocial.gov.co/VB eContent/NewsDetail.asp?ID=14774\&IDCompany=3 Acceso el 31 mayo de 2006.

28. Isaza CA, Osorio FJ, Mesa G, Moncada JC. Patrones de uso de antihipertensivos en 11.947 pacientes colombianos. Biomédica. 2002;22:476-85.

29. Grant R, Buse J, Meigs J. Quality of diabetes care in U.S. Academia Medical Centres. Diabetes Care. 2005;28:337-42.

30. Resnick H, Foster G, Bardsley J, Ratner R. Achievement of American Diabetes Association clinical practice recommendations among U.S. adults with diabetes, 1999-2002. Diabetes Care. 2006;29:531-7. 
31. Mangione C, Gerzoff R, Williamson D, Steers $\mathrm{N}$, Kerr E, Brown A, et al. The association between quality of care and the intensity of diabetes disease management programs. Ann Intern Med. 2006;145:107-16.

32. Ravnskov U. Glomerular, tubular and interstitial nephritis associated with non-steroidal antiinflammatory drugs. Evidence of a common mechanism. Br J Clin Pharmacol. 1999; 47:203-10.
33. Mas J. Atherothrombosis: management of patients at risk. Int J Clin Pract. 2005;59:407-14.

34. Yan Y, Phillips D. Aspirin response and failure in diabetic patients with cardiovascular disease. Curr Opin Pharmacol. 2005;5:190-7.

35. Evangelista V, Totani L, Rotondo S, Lorenzt R, Tognoni G, De Berardis G, et al. Prevention of cardiovascular disease in type 2 diabetes: how to improve the clinical efficacy of aspirin. Thromb Haemost. 2005;93:8-16.
36. Gómez J, Candás M, Fidalgo G, Armesto J, Calvo M, de Marino M, et al. Análisis del consumo de medicamentos utilizando indicadores de calidad en la prescripción. Aten Primaria. 2000;25:618-24

Manuscrito recibido el 11 de julio de 2006. Aceptado para publicación, tras revisión, el 28 de junio de 2007.

ABSTRACT Objectives. To identify prescription patterns for diabetes treatment among a group of individuals covered by Colombia's universal health care, the General System for Social Security in Health (SGSSS).

Antidiabetic drugs prescription patterns among a group of patients in Colombia

Methods. The study included 7308 patients covered by SGSSS who had diabetes mellitus type 1 or 2, were of either sex, any age, and had been undergoing treatment for at least three months (May-July 2005) in 19 cities in Colombia. A database was compiled from the medication usage records maintained by the dispensing pharmacy. Data were analyzed using SPSS 13.0 (SPSS Inc., Chicago, Illinois, United States of America). Student's $t$-test and analysis of variance (ANOVA) were employed to compare quantitative variables, and the chi-square test was applied to compare categorical variables.

Results. The patients' mean age was $60.7 \pm 12.7$ years. Women made up $56.6 \%$ of the group and had a mean age significantly higher than that of the men $(P<0.05)$. Fortyeight percent of the group had been prescribed one diabetes medication, while $52 \%$ had been prescribed from two to four. The medications prescribed were: biguanides $(67.5 \%)$, sulphonylureas $(64.9 \%)$, insulin $(23.5 \%)$, and thiazolidinediones $(0.1 \%)$. The most common oral combination-therapies were: glibenclamide and metformin $(n=2$ $847)$, metformin and insulin $(n=510)$, glibenclamide and insulin $(n=148)$, and metformin, insulin, and glibenclamide $(n=288)$. Of the total, $94.3 \%$ had comorbid conditions for which they had been prescribed medication: antihypertensive drugs (in $74.4 \%$ of the cases), anti-inflammatories $(61.5 \%)$, hypolipemiants $(45.5 \%)$, antiulcer medications $(21.0 \%)$, psychoactive drugs $(16.8 \%)$, antimicrobials $(14.4 \%)$, asthma medication $(5.3 \%)$, and salicylic acid $(2.8 \%)$. Prescriptions for comorbid conditions were more common among women than men $(95.6 \%$ vs. $92.7 \%, P<0.001)$. Undertreatment with certain medications (metformin, thiazolidinediones, alpha-glucosidase inhibitors, and salicylic acid), and overtreatment with others (antiulcer drugs), probably exist.

Conclusions. There are significant differences in oral therapies prescribed for diabetes across the 19 cities studied, but overall, prescription patterns are appropriate. Educational strategies should be developed to address those prescribing practices that are not appropriate, and the clinical results of the medications studied should be explored.

Key words Diabetes mellitus, hypoglycemic agents, pharmaceutical preparations, Colombia. 